\title{
Local lattice relaxations in random metallic alloys: Effective tetrahedron model and supercell approach
}

\author{
Ruban, Andrei; Simak, S.I.; Shallcross, S.; Skriver, Hans Lomholt
}

Published in:

Physical Review B Condensed Matter

Link to article, DOI:

10.1103/PhysRevB.67.214302

Publication date:

2003

Document Version

Publisher's PDF, also known as Version of record

Link back to DTU Orbit

Citation (APA):

Ruban, A., Simak, S. I., Shallcross, S., \& Skriver, H. L. (2003). Local lattice relaxations in random metallic alloys: Effective tetrahedron model and supercell approach. Physical Review B Condensed Matter, 67(21), 214302. https://doi.org/10.1103/PhysRevB.67.214302

\section{General rights}

Copyright and moral rights for the publications made accessible in the public portal are retained by the authors and/or other copyright owners and it is a condition of accessing publications that users recognise and abide by the legal requirements associated with these rights.

- Users may download and print one copy of any publication from the public portal for the purpose of private study or research.

- You may not further distribute the material or use it for any profit-making activity or commercial gain

- You may freely distribute the URL identifying the publication in the public portal 


\title{
Local lattice relaxations in random metallic alloys: Effective tetrahedron model and supercell approach
}

\author{
A. V. Ruban, ${ }^{1}$ S. I. Simak, ${ }^{2}$ S. Shallcross, ${ }^{2}$ and H. L. Skriver ${ }^{1}$ \\ ${ }^{1}$ Center for Atomic-scale Materials Physics and Physics Department, Technical University of Denmark, DK-2800 Lyngby, Denmark \\ ${ }^{2}$ Condensed Matter Theory Group, Physics Department, Uppsala University, Box-530, S-75121 Uppsala, Sweden
}

(Received 4 December 2002; published 20 June 2003)

\begin{abstract}
We present a simple effective tetrahedron model for local lattice relaxation effects in random metallic alloys on simple primitive lattices. A comparison with direct $a b$ initio calculations for supercells representing random $\mathrm{Ni}_{0.50} \mathrm{Pt}_{0.50}$ and $\mathrm{Cu}_{0.25} \mathrm{Au}_{0.75}$ alloys as well as the dilute limit of Au-rich $\mathrm{CuAu}$ alloys shows that the model yields a quantitatively accurate description of the relaxtion energies in these systems. Finally, we discuss the bond length distribution in random alloys.
\end{abstract}

DOI: 10.1103/PhysRevB.67.214302

PACS number(s): 64.90.+b, 75.47.Np

\section{INTRODUCTION}

In spite of the substantial progress made in understanding the structural properties of different materials in general, ${ }^{1}$ a quantitative description of lattice relaxation effects through simplified models still remains a problem. There is, however, a class of systems where a simple and accurate solution may be found. In alloys of the late transition and noble metals noncentral forces (or multiatom interactions) are quite small due to an almost empty $p$ band and a nearly fully occupied $d$ band. The local lattice relaxations caused by a specific alloy configuration on the lattice are therefore mainly determined by the atomic size mismatch of the alloy components and, as a result, one may expect to be able to derive a simple but quite accurate model for the relaxation energies involved.

In general, one may distinguish between three different types of lattice relaxations which may occur in metallic alloys due to a specific atomic configuration on the underlying lattice: (1) anisotropic lattice distortions leading to a change in the form of the unit cell, (2) isotropic or volume relaxations, and (3) local displacements of atoms from the ideal, underlying lattice positions. The anisotropic lattice distortions are caused by a reduction of the global symmetry of the underlying lattice due to a specific long range order. The energy of such relaxations can be quite large and may easily dominate the ordering energy itself. For instance, in the case of the CuAu- $L 1_{0}$ ordered alloy the energy of the tetragonal lattice distortion is only about $0.012 \mathrm{eV}$. However, in the case of the $Z 2$ "phase-separated"-like structure it reaches 0.143 $\mathrm{eV}^{2}$ In the case of $\mathrm{Z3}-\mathrm{Au}_{3} \mathrm{Cu}$ alloy the relaxation energy is $0.08 \mathrm{eV}$, and this is sufficient to make the $Z 3$ structure more stable than $L 1_{2}$ at $0 \mathrm{~K}^{2}$ At the same time, in a number of ordered phases such relaxations are either absent $\left(L 1_{2}, B 2\right.$, and $\left.D_{3}\right)$, or very small $\left(D \mathrm{O}_{22}, D \mathrm{O}_{23}\right.$, and so on) due to the symmetry.

If the global symmetry of the underlying lattice is preserved (on average in the case of random alloys), but the point group of different sites is much lower than that of the underlying lattice due to the local atomic configuration, then local displacements of atoms from the ideal (underlying primitive) lattice positions may occur. The energy of such local relaxations can also be quite large. For instance, according to the results of first-principles calculations for the so-called special quasirandom structures (SQS's), ${ }^{2}$ the energy of the local relaxations in random $\mathrm{Cu}_{0.75} \mathrm{Au}_{0.25}$ and $\mathrm{Cu}_{0.50} \mathrm{Au}_{0.50}$ alloys is about $0.05 \mathrm{eV}$, which is half of the value of the $L 1_{2}$ ordering energy, ${ }^{2}$ and therefore a quantitatively accurate theoretical description of the configurational energetics of $\mathrm{CuAu}$ alloys is impossible without a proper account taken of these lattice relaxation effects.

In contrast, the isotropic volume relaxations associated with the change of the atomic configuration on the lattice are usually very small: For instance, the volume relaxation energy due to the order-disorder phase transition in $\mathrm{Cu}_{0.75} \mathrm{Au}_{0.25}$ alloy is about $2 \mathrm{meV}$ for the theoretical change of the lattice spacing of $0.02 \AA$ obtained without short range order and local atomic relaxations in random alloy. ${ }^{3}$ Such a change of the lattice spacing is in fact of the order of the thermal expansion of the $\mathrm{Cu}_{3} \mathrm{Au}$ from $\mathrm{O} \mathrm{K}$ to ambient temperatures, and the commonly used approximations for the exchange-correlation energy do not provide a better accuracy for the enthalpies of formation of metallic alloys (which seems to be in itself related to the problem of the error in the equilibrium lattice spacing-see below).

Despite the fact that the lattice relaxations can be quite accurately obtained in first-principles calculations, their general account in statistical thermodynamic calculations for random and partially ordered alloys is still a problem. For instance, the most widely used mixed-space cluster expansion (MSCE) is not only cumbersome, but also has illdefined limits: The so-called constituent strain energy term prescribes a constant value for the "strain" energy of all concentration waves in a given direction. That is, according to this model the "strain" energies of, for instance, the $L 1_{2}$ and $Z 3$ ( $Z 1)$ structures are the same, since their atomic configurations are described by the concentration wave going in the [001] direction. However, there can be no relaxations except isotropic volume relaxations in the $L 1_{2}$ structure, while the tetragonal distortions of the $Z 3$ structure could be quite substantial, as indeed is the case of the $\mathrm{Au}_{3} \mathrm{Cu}$ alloy mentioned above.

An effort has been made in Ref. 4 to use another model for the relaxation energy which, as in the concentration wave formalism, is determined in reciprocal space, but now the relaxation interactions have been chosen to depend exclusively on the absolute value of the wave number of the con- 
centration wave. Nevertheless, the whole scheme, applied to the $\mathrm{CuAu}$ system, turned out to be quite complicated and sensitive: It seems that it is necessary to do a lot of quite accurate calculations of different ordered structures to get a reasonable convergence of the expansion.

The main reason for the problems with the MSCE and the approach of Ref. 4 is an attempt to account for the whole variety of lattice relaxation effects by using an analytically simple term taken from the static displacement formalism of Krivoglaz ${ }^{5}$ and Khachaturyan, ${ }^{6}$ which can in fact only be obtained due to some very specific assumptions about force constants in the system. ${ }^{5}$ However, there is no a priori need to take into consideration all kinds of relaxation effects if not all of them are simultaneously important in the description of the phenomena of interest. For example, in the simulations of order-disorder phase transitions in binary alloys, it makes no sense to include in the Hamiltonian a term which accounts for inhomogeneous lattice distortions or the strain energy of long-period superstructures $A_{m} B_{n}$, if such structures are known not to appear and the system does not undergo an inhomogeneous lattice distortion during the transition. What should be taken into consideration in such a case, however, is the local lattice relaxations.

Just for this particular purpose we suggest in the present paper an alternative effective tetrahedron model (ETM), which greatly simplifies the inclusion of the local lattice relaxation effects in statistical thermodynamic simulations. Although its application is restricted to metallic alloys without strong noncentral forces and anisotropic lattice distortions, it is extremely simple and durable, and, it appears, quite accurate. The ETM is based on the assumption that the local lattice relaxations in alloys are the result of the local volume relaxations. It is in fact similar to the effective-cluster volume scheme proposed by Amador et al. ${ }^{7}$ As we demonstrate in this paper, being extremely simple and easily adaptable to configurational thermodynamic simulations, it yields quantitatively accurate energetics of the local relaxations in late transition metal alloys and nobel metal alloys.

\section{EFFECTIVE TETRAHEDRON MODEL}

A starting point for the model is the division of the Hamiltonian of a binary $A_{1-{ }_{c}} B_{c}$ alloy into two parts (although the model may easily be generalized to the case of multicomponent alloys),

$$
H\left(\Omega_{0}\right)=E_{0}\left(\Omega_{0}\right)+E_{r e l}\left(\Omega_{0}\right),
$$

where the first term, $E_{0}\left(\Omega_{0}\right)$, is the energy of the alloy with a given atomic configuration (and concentration) on the ideal primitive underlying lattice and the second term, $E_{r e l}\left(\Omega_{0}\right)$, the local relaxation energy, i.e., the energy which the system gains by fully relaxing all atomic positions. $\Omega_{0}$ is the equilibrium volume per atom for a given alloy composition (we will neglect its configuration dependence, since in many cases it is quite small, as has been discussed in the Introduction).

Let us assume next that the relaxation energy of the smallest cluster in the underlying alloy lattice is a function mainly of the change in volume of that cluster. If this is the case then the total relaxation energy of an alloy may be expressed as a sum over such clusters. Since the smallest cluster having non-zero volume is a tetrahedron the relaxation energy of a binary $A_{c} B_{1-c}$ alloy can be written in the form

$$
E_{r e l}=\frac{1}{4} \sum_{i, j, k, l} V_{r e l}\left(c_{i}, c_{j}, c_{k}, c_{l}\right)
$$

where $V_{r e l}$ is the relaxation interaction which is a function of the occupation numbers $\left\{c_{i}\right\}=c_{i}, c_{j}, c_{k}$, and $c_{l}\left(c_{i}=1\right.$ if site $i$ is occupied by a $B$ atom; otherwise it is 0 ) for the corresponding tetrahedron verticies $i, j, k$, and $l$. In the case of the fcc lattice the tetrahedron of the nearest neighbors is the smallest one. In this case $V_{r e l}$ depends only on the composition of the tetrahedron, which can be $A_{4}, A_{3} B, A_{2} B_{2}$, $A B_{3}$, and $B_{4}$, since all configurations are equivalent for a fixed number of $A$ and $B$ atoms in the tetrahedron. Note, that this form may be transformed into the usual form of the Ising-type Hamiltonian, used in statistical thermodynamic simulations (see the Appendixes), although it is also easily implemented directly in the Monte Carlo method.

Given this definition of the relaxation energy, we now need to find a method to obtain $V_{r e l}$ from first-principles calculations. A simple way to proceed is the following (which conceptually is similar to the well-known average $t$-matrix approximation in alloy theory ${ }^{8}$ ). Let us consider a random alloy given by "effective medium" atoms in the ideal crystal lattice positions. Such an alloy is, for instance, given by the coherent potential approximation. ${ }^{8-10}$ Then $V_{r e l}$ is the relaxation energy of a given tetrahedron of real atoms embedded in this rigid effective medium. Since it is hardly possible to obtain $V_{\text {rel }}$ defined in this way from firstprinciples calculations, we follow another approach. We begin by noting that certain ordered structures consist of only one type of tetrahedron. In the case of an fcc binary alloy they are: fcc- $A$ for the $A_{4}$ tetrahedron, $L 1_{2}-A_{3} B$ for $A_{3} B$, $L 1_{2}-A B_{3}$ for $A B_{3}, L 1_{0}$ for $A_{2} B_{2}$ and fcc- $B$ for $B_{4}$. In this way we may calculate the relaxation interactions as the volume relaxation energy of some special $\alpha$ structures:

$$
V_{r e l}\left(\left\{c_{i}\right\}\right)=E^{\alpha}\left[\Omega_{r e l}\left(\left\{c_{i}\right\}\right)\right]-E^{\alpha}\left(\Omega_{0}\right),
$$

where $E^{\alpha}$ is the total energy per atom of an ordered $\alpha$ structure associated with a given tetrahedron configuration $\left\{c_{i}\right\}$, $\Omega_{0}$ the volume of the unrelaxed tetrahedron, and $\Omega_{r e l}\left(\left\{c_{i}\right\}\right)$ the volume of the fully relaxed tetrahedron with configuration $\left\{c_{i}\right\}$ embedded in the effective medium.

In the case of binary bcc alloys, where the smallest tetrahedron is formed by 4 sides connecting the nearest neighbor atoms and two sides connecting the next nearest neighbor atoms, one has actually two nonequivalent $A_{2} B_{2}$ tetrahedra: one is present in the $B 2$ structure and the other one in the $B 32$ structure. The $A_{3} B$ and $A B_{3}$ tetrahedra in the bcc structure are given by the $D_{3}$ structure and pure $A_{4}$ and $B_{4}$ tetrahedra by the bcc structure.

\section{HARMONIC SPRING MODEL FOR $A-A, A-B$, AND $B-B$ BOND LENGTHS}

The volume of the fully relaxed tetrahedron, $\Omega_{r e l}\left(\left\{c_{i}\right\}\right)$, may be obtained from the corresponding bond lengths which 


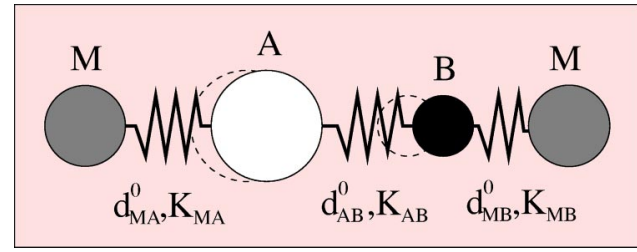

FIG. 1. (Color online) Schematic picture of the harmonic spring model: The positions of effective medium atoms $(M)$ are fixed, while $A$ and $B$ atoms are relaxed according to the initial equilibrium bond lengths and spring constants.

form the tetrahedron sides. The latter can be determined in a simple harmonic spring model, which takes into consideration only the interactions along a given $A(B)-B(A)$ bond. Let us consider the chain $M-A-B-M$, where $M$ are the effective medium atoms. The positions of the effective medium atoms are assumed to be fixed, while the positions of the $A$ and $B$ atoms are allowed to relax according to the initial equilibrium bond lengths, $d_{X Y}^{0}$ and spring constants, $K_{X Y}$ for the individual pair of $X$ and $Y$ atoms (see Fig. 1).

To solve the spring model for every pair of atoms one needs to know the equilibrium lengths and spring constants of the following bonds: $A-A, A-B, B-B, M-M, M-A$, and $M-B$. It is clear that the parameters for the $A-A, B-B$, and $M-M$ bonds are given by the ground state properties of the pure $A$ and $B$ components and of the random alloy $A_{1-c} B_{c}$ on the corresponding underlying crystal lattice. For the $A-B$ bond we suggest using the simplest ordered $A B$ structure having the lowest value of the Warren-Cowley short range order parameter at the first coordination shell. This is the $L 1_{0}$ ordered structure in the case of fcc alloys.

The parameters for the $M-A$ and $M-B$ bonds can be determined by an interpolation of the corresponding parameters from the data for the $A-A, A-B$, and $M-M$ bonds. The simplest choice is just Zen's law (Vegard's law may be used instead) for the bond lengths, and a simple average of the spring constants, i.e.,

$$
\begin{gathered}
d_{M A}^{0}=\left[\frac{\left(d_{M M}^{0}\right)^{3}+\left(d_{A A}^{0}\right)^{3}}{2}\right]^{1 / 3}, \\
K_{M A}=\frac{1}{2}\left(K_{M M}+K_{A A}\right),
\end{gathered}
$$

where $d_{M A}^{0}$ and $K_{M A}$ are the "equilibrium" length and the spring constant between of the $M-A$ bond.

By solving the spring model one finally gets the following expressions for the equilibrium $A-A(B-B)$ and $A-B$ interatomic distances in the tetrahedron:

$$
\begin{gathered}
d_{A A}=d_{A A}^{0}+\frac{2\left(d_{M M}^{0}-d_{A A}^{0}\right)}{2 \frac{B_{A}}{B_{M}}+1}, \\
d_{A B}=d_{A B}^{0}+\frac{2 d_{M M}^{0}-\frac{1}{2}\left(d_{A A}^{0}+2 d_{A B}^{0}+d_{B B}^{0}\right)}{\frac{B_{A B}}{B_{M B}}+\frac{B_{A B}}{B_{M A}}+1},
\end{gathered}
$$

where we have used the fact that the spring constant, $K_{X Y}$, is proportional to the corresponding bulk modulus $B_{X Y}$.

\section{DETAILS OF THE CALCULATIONS}

The parameters of the spring model and ETM have been obtained in Korringa-Kohn-Rostoker (KKR) self-consistent density functional calculations in the atomic sphere approximation (ASA), ${ }^{11}$ which has been corrected by the use of both the muffin-tin correction for the Madelung energy, ${ }^{12}$ needed for obtaining an accurate description of ground state properties in the ASA, and the multipole moment correction to the Madelung potential and energy ${ }^{11}$ which significantly improves the accuracy by taking into consideration the nonspherical part of polarization effects. Although we have used the local density approximation (LDA) with Perdew and Wang parametrization of the exchange-correlation potential, ${ }^{13}$ the total energies have been calculated in three different approximations for the exchange-correlation energy: LDA, ${ }^{13}$ local Airy gas, ${ }^{14}$ and generalized gradient approximation (GGA). ${ }^{15}$ The partial waves in the KKR-ASA calculations have been expanded up to $l_{\max }=3$ inside atomic spheres, although the multipole moments of the electron density have been determined up to $l_{\max }^{M}=6$ for the multipole moment correction to the Madelung energy. The core states have been recalculated after each iteration.

The ground state properties of random alloys have been obtained in density functional theory single-site KKR-ASA coherent potential approximation (CPA) calculations with the Coulomb screening potential, $V_{s c r}^{i}$, and energy $E_{s c r}:{ }^{16}$

$$
\begin{gathered}
V_{s c r}^{i}=e^{2} \alpha \frac{q_{i}}{S} \\
E_{s c r}=-e^{2} \frac{\beta}{2 S} \alpha \sum_{i} c_{i} q_{i}^{2}
\end{gathered}
$$

where $q_{i}$ and $c_{i}$ are the net charge of the atomic sphere and concentration of the $i$ th alloy component, $S$ the Wigner-Seitz radius, and $\alpha$ and $\beta$ are screening constants determined from supercell calculations using the locally self-consistent Green's function method (LSGF). ${ }^{17}$ For fcc-NiPt and fcc$\mathrm{CuAu}$ alloys the $\alpha$ and $\beta$ screening constants were 0.74 and 1.16 , respectively.

In Table I we show our results for the equilibrium WignerSeitz radii and bulk moduli of pure fcc $\mathrm{Ni}, \mathrm{Cu}, \mathrm{Au}$ and $\mathrm{Pt}$ metals as well as ordered $L 1_{0}-\mathrm{CuAu}$ and NiPt alloys obtained by the KKR-ASA-(CPA) method in the different approximations for the exchange-correlation energy. It is obvious that none of these approximations provides the best overall description of the ground state properties of all the systems: The LDA being good for $5 d$ metals substantially underestimates the Wigner-Seitz radius for $3 d$ metals, and subsequently overestimates their bulk modulus of these metals. The GGA is, conversely, quite good for the $3 d$ metals, although it overestimates significantly the Wigner-Seitz radius of $5 d$ metals and underestimates their bulk modulii. We would like to point out that this trend is general for the late transition metals (also see Ref. 18). 
TABLE I. Equilibrium Wigner-Seitz radii and bulk moduli (in parentheses), obtained in the $\operatorname{KKR}-\mathrm{ASA}(+\mathrm{M})$ calculations. The Wigner-Seitz radii in atomic units and the bulk moduli in Mbar.

\begin{tabular}{lcccc}
\hline \hline System & LDA & LAG & GGA & Expt. \\
\hline fcc-Ni & $2.516(2.66)$ & $2.552(2.30)$ & $2.584(1.90)$ & $2.60(1.87)$ \\
fcc-Cu & $2.585(1.96)$ & $2.630(1.63)$ & $2.666(1.37)$ & $2.66(1.42)$ \\
fcc-Pt & $2.871(3.13)$ & $2.899(2.75)$ & $2.926(2.51)$ & $2.89(2.78)$ \\
fcc-Au & $2.990(1.94)$ & $3.034(1.57)$ & $3.071(1.37)$ & $2.89(1.73)$ \\
\hline$L 1_{0}-\mathrm{CuAu}$ & $2.810(1.96)$ & $2.853(1.61)$ & $2.889(1.43)$ & $2.85(-)$ \\
$L 1_{0}-\mathrm{NiPt}$ & $2.715(2.90)$ & $2.747(2.53)$ & $2.775(2.32)$ & $2.75(-)$ \\
rand-NiPt & $2.723(2.81)$ & $2.756(2.46)$ & $2.784(2.26)$ & \\
\hline \hline
\end{tabular}

The direct first-principles calculations of the local lattice relaxations in random fcc and bcc alloys modeled by supercells have been performed by the Vienna ab initio simulation package (VASP), which is described in detail in Refs. 19 and 20. In these calculations we have assumed the local lattice relaxations are well "screened" at distances beyond the the first three or four coordination shells, and therefore we have used supercells of quite moderate size, which are called SQSs following the terminology of Ref. 21. The atomic distribution correlation functions, or the Warren-Cowley shortrange order (SRO) parameters were as in the real random alloy in the first several coordination shells.

The calculations were performed in a plane-wave basis, utilizing fully nonlocal Vanderbilt-type ultrasoft pseudopotentials $\mathrm{s}^{22}$ which allow the use of a moderate cutoff for the construction of the plane-wave basis for the transition metals. ${ }^{23}$ The integration over the Brillouin zone was done on special $k$-points determined according to the MonkhorstPack scheme. ${ }^{24}$ All necessary convergence tests were performed, and generally the required total energy convergence (within $0.2 \mathrm{mRy} / \mathrm{atom}$ ) was reached for $4-63 k$ points in the irreducible wedge of the Brillouin zone depending on the structure and total number of atoms. All the KKR-ASA and VASP calculations are scalar relativistic.

\section{RESULTS}

\section{A. $\mathrm{Ni}_{0.50} \mathbf{P t}_{0.50}$ random alloy}

We consider first the local lattice relaxations in the random $\mathrm{Ni}_{0.50} \mathrm{Pt}_{0.50}$ alloy, where the local lattice relaxations are quite pronounced due to the substantial size mismatch of the alloy components. The GGA nearest neighbor interatomic distance in the random alloy on the locally unrelaxed lattice given by the KKR-ASA-CPA calculations is $2.681 \AA$. The relaxed distances obtained in the supercell full potential (FP) calculations by VASP, are shown in Fig. 2. It is obvious that there is a substantial dispersion of bond lengths for all three different pairs, and that the Pt-Pt bond length can be much less than that of the alloy average, as well the Ni-Ni bond being much longer. Nevertheless, the average values of the bond lengths follow the expected trend: They increase in the sequence of Ni-Ni, Ni-Pt and Pt-Pt: 2.646, 2.671, and $2.741 \AA$.

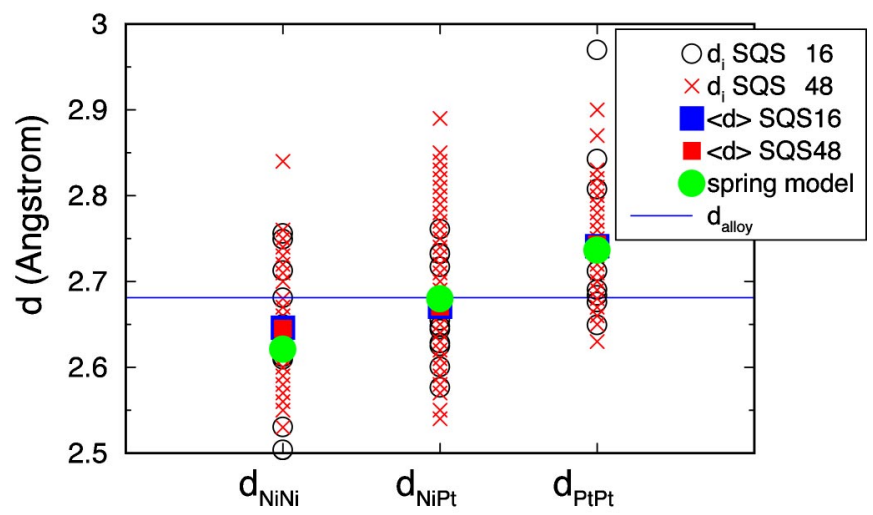

FIG. 2. (Color online) Nearest neighbor bond lengths in a "quasirandom" $\mathrm{Ni}_{0.50} \mathrm{Pt}_{0.50}$ alloy.

As one can see in Fig. 2 the spring model described in the previous section works quite well for the average bond lengths, although it does not reproduce a certain asymmetry in the relaxations: The average local relaxations of the $\mathrm{Ni}-\mathrm{Ni}$ bonds being measured from the equilibrium bond lengths in the pure element is greater than that of Pt-Pt. This is, in fact, a consequence of using the harmonic approximation to describe bond interactions in the spring model. In real systems, however, the interatomic bonding is anharmonic: It is usually much easier to expand the lattice from its equilibrium value than to squeeze it, an effect which is also the origin of the thermal lattice expansion. However, as will be demonstrated below, it turns out that the harmonicity of the spring model allows one to describe specific local environment effects in the ETM.

Using the values of the bond lengths for individual pairs, one can now determine the volume of the corresponding relaxed tetrahedra in the alloy and calculate the relaxation interactions from Eq. (3). Such interactions for the $\mathrm{Ni}_{0.50} \mathrm{Pt}_{0.50}$ alloy are given in Table II. As one can expect the smallest value of the relaxation energy corresponds to the tetrahedron whose composition coincides with that of the alloy. It is, in fact, almost zero in this case, which provides a proper limit for the local lattice relaxation energy: in the completely ordered $\left(L 1_{0}\right)$ state, the energy of the local lattice relaxations should vanish. The greater the difference between the tetrahedron and alloy compositions, the greater the relaxation energy is. Moreover, one may also notice that there is a certain asymmetry in the value of relaxation interactions: The relaxation energy for the $\mathrm{Pt}_{4}$ is about $50 \%$ greater than that for $\mathrm{Ni}_{4}$. Such an asymmetry is again the result of the anharmonic behavior of the equation of state.

The local lattice relaxation energy of a random $A_{1-c} B_{c}$ alloy, without short-range order effects, will be given by

TABLE II. Relaxation interactions in $\mathrm{Ni}_{0.50} \mathrm{Pt}_{0.50}$ alloy (in $\mathrm{meV})$.

\begin{tabular}{lccccc}
\hline \hline & $V_{N_{4}}$ & $V_{N i_{3} P t}$ & $V_{N_{2} P_{2}}$ & $V_{N i P t_{3}}$ & $V_{P_{4}}$ \\
\hline LDA & -188.3 & -58.5 & $\sim 0.0$ & -59.2 & -270.3 \\
LAG & -159.6 & -49.9 & $\sim 0.0$ & -50.6 & -235.4 \\
GGA & -156.1 & -44.9 & $\sim 0.0$ & -49.3 & -210.3 \\
\hline \hline
\end{tabular}


TABLE III. The local relaxation in a random $\mathrm{Ni}_{0.50} \mathrm{Pt}_{0.50}$ alloy (in meV/atom).

\begin{tabular}{lccc}
\hline \hline Method & LDA & LAG & GGA \\
\hline ETM & -58 & -50 & -46 \\
CWM (Ref. 25) & -54 & & \\
SQS-16 & -58 & & -46 \\
SQS-48 & -58 & & -45 \\
\hline \hline
\end{tabular}

$$
\begin{aligned}
E_{r e l}= & (1-c)^{4} V_{A_{4}}+4 c(1-c)^{3} V_{A_{3} B}+6 c^{2}(1-c)^{2} V_{A_{2} B_{2}} \\
& +4 c^{3}(1-c) V_{A B_{3}}+c^{4} V_{B_{4}} .
\end{aligned}
$$

In Table III we compare the ETM, the cluster expansion results by Lu et al., ${ }^{25}$ and our direct 16- and 48-atom supercell GGA calculations for $\mathrm{Ni}_{0.50} \mathrm{Pt}_{0.50}$. Although all these results have been obtained by using entirely different methods, the agreement between them is fairly good. This indicates that the energy of the local lattice relaxations is quite insensitive to the details of the atomic displacements.

There is, however, an important point here: while the energy of the local lattice relaxations is insensitive to the details of the model, it is nevertheless quite affected by the choice of approximation for the exchange-correlation energy. One can see from the results presented in Table III that the difference between LDA and GGA results is about $0.01 \mathrm{eV}$, which is approximately $20 \%$ of the the value of the relaxation energy. At the same time, as has already been mentioned, none of the existing approximations for the exchange-correlation energy provides an accurate description of the ground state properties of both $\mathrm{Ni}$ and $\mathrm{Pt}$.

\section{B. $\mathrm{Cu}-\mathrm{Au}$ random alloys and the dilute limit of $\mathrm{Cu}$ in $\mathrm{Au}$}

As already discussed in Sec. I, lattice relaxation effects play an important role in the phase equilibria of the $\mathrm{CuAu}$ system. This system is also very interesting for studying lattice relaxations since experiment ${ }^{26}$ and first-principles fullpotential calculations ${ }^{2,4}$ (also see the effective medium theory ${ }^{27}$ results) report the effect of a "loosening" of the $\mathrm{Cu}-\mathrm{Cu}$ bond in $\mathrm{Au}$-rich random alloys leading to a specific crossover when, with increasing concentration of $\mathrm{Au}$, the average $\mathrm{Cu}-\mathrm{Cu}$ bond length becomes greater than that of $\mathrm{Cu}$ $\mathrm{Au}$.

Note, however, that in fact neither the experimental data $^{26}$ nor the theoretical results of Refs. 2 and 4 provide a completely convincing picture of the existence of such a phenomenon. First of all, they are not in quantitative agreement with each other: According to the experimental data the crossover occurs when the content of $\mathrm{Au}$ in the random alloy exceeds about 86 at. \%, although the theoretical calculations show that it has already happened in the random $\mathrm{Cu}_{0.25} \mathrm{Au}_{0.75}$ alloy where the average $\mathrm{Cu}-\mathrm{Cu}$ bond length is much greater than that of $\mathrm{Cu}-\mathrm{Au}$. Second, the uncertainty in the experimental results for the $\mathrm{Cu}-\mathrm{Cu}$ bond length is too large to make an accurate prediction of its value relative to the $\mathrm{Cu}-\mathrm{Au}$ bond length. At the same time, the eight-atom supercell (SQS-14 ${ }_{a}$ ) used in the first-principles

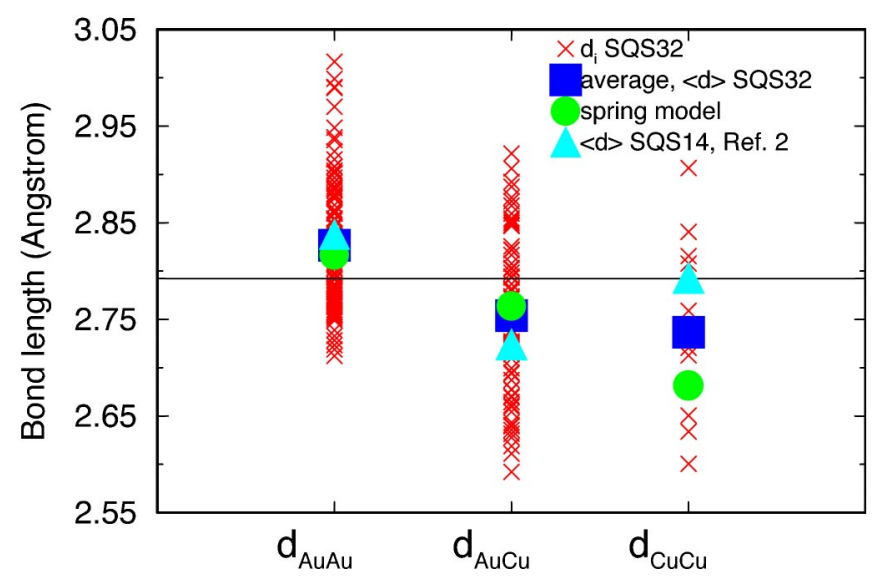

FIG. 3. (Color online) Nearest neighbor bond lengths in a "quasirandom" $\mathrm{Au}_{0.75} \mathrm{Cu}_{0.25}$ alloy.

calculations $^{2,4}$ could be too small to reproduce correctly the statistics of bond length distributions in the random alloy.

To check the bond length distribution in Au-rich $\mathrm{Cu}-\mathrm{Au}$ random alloys we have performed FP VASP calculations for a 32-atom supercell of the $\mathrm{Cu}_{0.25} \mathrm{Au}_{0.75}$ alloy described above, which produces a much better representation of the random alloy than the SQS-14 ${ }_{a}(b)$ cell used in Ref. 2. The relaxed bond lengths of the SQS-32 are presented in Fig. 3. As in the case of the NiPt random alloy, there is a huge dispersion of all possible types of pairs. However, the average interatomic distances follow the usual behavior: $d_{A u A u}$ $>d_{A u C u}>d_{C u C u}$ in accordance with the experimental data. ${ }^{26}$ The results of the spring model, which in fact yields a quite reasonable representation of the average bond lengths, also follow this trend. However, as in the case of the $\mathrm{Ni}_{0.50} \mathrm{Pt}_{0.50}$ random alloy, the average $\mathrm{Cu}-\mathrm{Cu}$ bond length is underestimated due to the use of the harmonic approximation.

To investigate this problem further we have calculated by the FP-VASP method the local relaxations in the dilute limit of Au-rich alloys: a single $\mathrm{Cu}$ impurity and a pair of $\mathrm{Cu}$ nearest neighbor atoms in pure Au. A 32-atom supercell has been used in the case of a single impurity and a 72-atom supercell for the pair of $\mathrm{Cu}$ atoms. We have again used the LDA, which provides the best ground state properties of pure Au. The FP-VASP result for the lattice spacing is $4.0633 \AA$, which corresponds to $2.8732 \AA$ for the nearest neighbor $\mathrm{Au}-\mathrm{Au}$ bond length. In the case of a single $\mathrm{Cu}$ impurity the $\mathrm{Cu}-\mathrm{Au}$ bond length turned out to be $2.8254 \AA$ (a reduction of about $1.66 \%$ ).

In Fig. 4 we show the atomic displacements of $\mathrm{Cu}$ and $\mathrm{Au}$ atoms for the pair of $\mathrm{Cu}$ atoms in $\mathrm{Au}$. The $\mathrm{Cu}$ atoms are in the (001) plane, and their positions as well as the positions of the $\mathrm{Au}$ atoms in this plane are shown by solid symbols. The $\mathrm{Au}$ atoms in the next (001) plane are shown by dotted symbols. In this way every square of nearest neighbors in the figure corresponds to a tetrahedron of nearest neighbors in the underlying fcc structure. The indexes of the Au atoms label the nonequivalent positions. The final interatomic distances are given in Table IV together with the average $\mathrm{Cu}-\mathrm{Au}$ distance in this case.

The $\mathrm{Cu}-\mathrm{Cu}$ bond length in the dilute limit is actually a little bit greater than that of the average $\mathrm{Cu}-\mathrm{Au}$ bond for the 


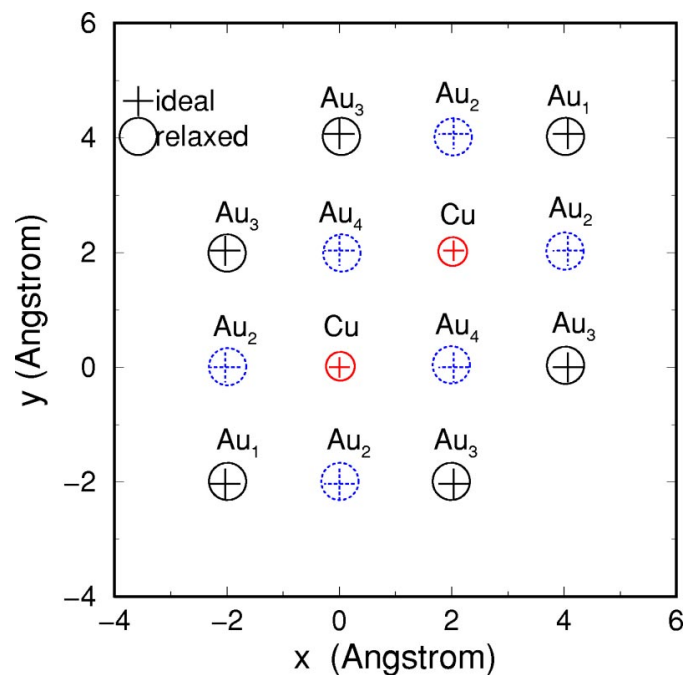

FIG. 4. (Color online) A view of the local relaxations of $\mathrm{Au}$ atoms around a pair of the nearest neghbor $\mathrm{Cu}-\mathrm{Cu}$ atoms in fcc $\mathrm{Au}$. Due to the chosen geometry of the 72-atom supercell all the relaxations occur in the (001) plane, shown in the figure. Atoms in the plane are shown by symbols drawn by solid lines, and atoms in the next (001) plane by dotted lines. Numbers mark equivalent $\mathrm{Au}$ atoms.

pair of $\mathrm{Cu}$ atoms, but it is still less than the $\mathrm{Cu}-\mathrm{Au}$ bond length in the case of a single $\mathrm{Cu}$ impurity. In the dilute limit of the $\mathrm{Au}_{1-c} \mathrm{Cu}_{c}$ random alloy, however, the last case will be dominating since the probability of having two $\mathrm{Cu}$ atoms as the nearest neighbors is $c^{2}$, even without taking into account the specific SRO effects at the first coordination shell in the $\mathrm{Cu}$-Au system, which must decrease even more this value, while it is only $c(1-c)$ in the case of a single impurity.

In other words, our supercell calculations do not confirm the existance of the crossover for $\mathrm{Cu}-\mathrm{Cu}$ and $\mathrm{Cu}-\mathrm{Au}$ bond lengths in random $\mathrm{Cu}-\mathrm{Au}$ alloys: The average $\mathrm{Cu}-\mathrm{Au}$ bond length will never be less than that of the average $\mathrm{Cu}-\mathrm{Cu}$. Of course, one should notice that the actual atomic displacements are only about $2 \%$ of the unrelaxed bond length.

The pair of $\mathrm{Cu}$ atoms in $\mathrm{Au}$ is a very interesting case since it shows how the model is able to reproduce some specific effects connected with the existence of a quite large dispersion of the bond lengths and at the same time also make a reasonable partition of the $E_{r e l}$ onto the corresponding inter-

TABLE IV. $\mathrm{Cu}-\mathrm{Cu}$ and $\mathrm{Cu}-\mathrm{Au}$ bond length distribution for the $\mathrm{CuCu}$ pair in pure $\mathrm{Au}$. The relative change of the bond length is given in parentheses.

\begin{tabular}{lcl}
\hline \hline $\begin{array}{l}\text { Type of bond } \\
\mathrm{Cu}-\mathrm{Cu}\end{array}$ & $\begin{array}{c}\text { Number } \\
\mathrm{Cu}-\mathrm{Au}\end{array}$ & $\begin{array}{l}\text { Bond length }(\AA) \\
2.8230(1.75 \%)\end{array}$ \\
$\mathrm{Cu}-\mathrm{Au}_{2}$ & 2 & $2.8402(1.15 \%)$ \\
$\mathrm{Cu}-\mathrm{Au}_{3}$ & 8 & $2.8329(1.40 \%)$ \\
$\mathrm{Cu}-\mathrm{Au} u_{4}$ & 4 & $2.8201(1.85 \%)$ \\
\hline $\mathrm{Cu}-\mathrm{Au}\rangle$ & 8 & $2.7671(3.69 \%)$ \\
\hline \hline
\end{tabular}

TABLE V. The local relaxation energy (LDA) in random $\mathrm{CuAu}$ alloys (in meV/atom).

\begin{tabular}{lccc}
\hline \hline Method & $\mathrm{Cu}_{0.75} \mathrm{Au}_{0.25}$ & $\mathrm{Cu}_{0.50} \mathrm{Au}_{0.50}$ & $\mathrm{Cu}_{0.25} \mathrm{Au}_{0.75}$ \\
\hline ETM & -51 & -52 & -36 \\
SQS-8,SQS-14 & -46 & -54 & -34 \\
$\quad$ Ref. 2) & & & -36 \\
SQS-32 & & & -36 \\
\hline \hline
\end{tabular}

actions. Let us consider the $\mathrm{Cu}-\mathrm{Au}$ bonds. There are four different $\mathrm{Cu}-\mathrm{Au}$ bond lengths presented in Table IV. The shortest $\mathrm{Cu}-\mathrm{Au}$ bond, which is, by the way, substantially shorter than the $\mathrm{Cu}-\mathrm{Cu}$ one, is between $\mathrm{Cu}$ and $\mathrm{Au}_{4}$ atoms. This is apparently due to specific positions of the $\mathrm{Au}_{4}$ atoms with respect to the $\mathrm{Cu}$ pair: In contrast to the rest of the $\mathrm{Au}$ atoms they have both $\mathrm{Cu}$ atoms as the nearest neighbors. That is, the large contraction of $\mathrm{Cu}-\mathrm{Au}_{4}$ bond is due to the presence of another $\mathrm{Cu}-\mathrm{Au}_{4}$ for the same $\mathrm{Au}$ atom, and thus it can be viewed as a multisite effect.

On the other hand, this can be also described as a local volume effect, since the $\mathrm{Au}_{4}-\mathrm{Cu}$ bond belongs to the $\mathrm{Cu}_{2} \mathrm{Au}_{2}$ tetrahedron of nearest neighbors, the only one which consists of two $\mathrm{Cu}$ atoms. There is in fact a quite interesting point about the ETM here. In the real alloy the $\mathrm{Cu}_{2} \mathrm{Au}_{2}$ tetrahedron has the smallest volume due the shortest $\mathrm{Cu}-\mathrm{Au}_{4}$ bonds. In the ETM it also has the smallest volume, but the reason is different: It is due to the shortest $\mathrm{Cu}-\mathrm{Cu}$ bond, whose contraction is quite overestimated by the harmonic approximation used in the spring model. That is, such an overestimation is very important, since it allows the ETM to "mimic" the appearance of very short $\mathrm{Cu}-\mathrm{Au}$ bonds in the $\mathrm{Cu}_{2} \mathrm{Au}_{2}$ tetrahedron, and thereby to produce the correct contribution to the local lattice relaxation energy.

This can be easily demonstrated, since in the ETM the relaxation energy of the $\mathrm{Cu}$ pair is $E_{r e l}^{\mathrm{CuCu}}=6 V_{\mathrm{Au}} \mathrm{Cu}$ $+V_{\mathrm{Au}_{2} \mathrm{Cu}_{2}}$ while that of a single $\mathrm{Cu}$ impurity is $E_{\text {rel }}^{\mathrm{Cu}}$ $=4 V_{A u_{3} C u}$, i.e., the later does not contain the $V_{\mathrm{Au}_{2} \mathrm{Cu}}$ interaction, which correspons to the $\mathrm{Cu}_{2} \mathrm{Au}_{2}$ tetrahedron. Therefore, if the ETM reproduces correctly both energies, for the single impurity and the pair impurity, it provides qualitatively correct partition of the relaxation energy in terms of the effective relaxation interactions. Indeed, the ETM value for the relaxation energy of a $\mathrm{Cu}$ impurity in $\mathrm{Au}$ is $-0.124 \mathrm{eV}$, which is in very good agreement with direct supercell calculations, $-0.12 \mathrm{eV}$, and at the same time the ETM relaxation energy for the pair of $\mathrm{Cu}$ atoms is $-0.304 \mathrm{eV}$ which is again in fairly good agreement with the VASP result, $-0.34 \mathrm{eV}$.

Finally, in Table V we compare the ETM and supercell results for the local lattice relaxation energies in random $\mathrm{Cu}_{0.75} \mathrm{Au}_{0.25}, \mathrm{Cu}_{0.50} \mathrm{Au}_{0.50}$ and $\mathrm{Cu}_{0.25} \mathrm{Au}_{0.75}$ alloys. The agreement is very good, even between SQS-14 $a(b)$ and SQS-32 supercell calculations, despite the fact that they produce a quite different average picture of the local lattice relaxations (Fig. 3). Such an insensitivity to the geometry of the relaxations is a clear indication that the local atomic relaxations in these alloys, as in the case of $\mathrm{Ni}_{0.50} \mathrm{Pt}_{0.50}$, is mostly the local volume relaxation effect. 


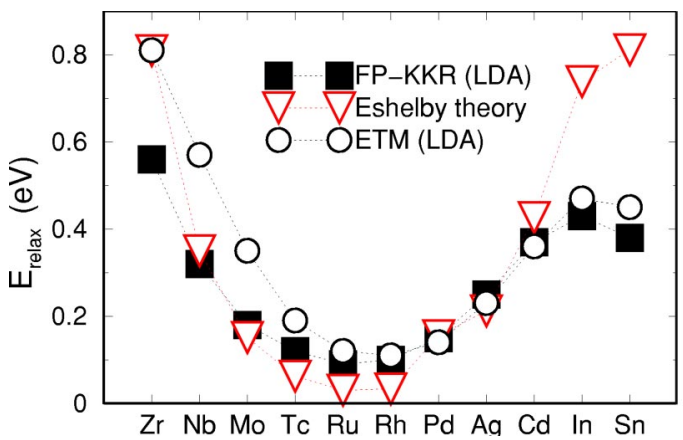

FIG. 5. Relaxation energies for the $4 d$ and $5 s p$ impurities in $\mathrm{Cu}$.

\section{Relaxation energies of $5 s p$ - and $4 d$ impurities in $\mathrm{Cu}$}

The fact that the volume effect dominates the energetics of local lattice relaxations for $3 d, 4 d, 4 s p$, and $5 s p$ impurities in $\mathrm{Cu}$ has in fact already been demonstrated in Ref. 28. Here it was shown that the relaxation energy scales rather well as the difference between the Wigner-Seitz radii of the host and impurity. On this basis a simple model was proposed that showed good agreement with calculations performed using both effective medium theory ${ }^{28}$ and the fullpotential Korringa-Kohn-Rostoker Green's function method. ${ }^{29}$ It is therefore very interesting to check how the ETM works here. The relaxation energy of a single impurity can be obtained as a partial molar quantity ${ }^{30}$ in the following way:

$$
E_{r e l}^{i m p}=\left.\frac{\partial E_{r e l}\left(A_{1-c} B_{c}\right)}{\partial c}\right|_{c=0}=V_{A_{4}}+4 V_{A_{3} B}=4 V_{A_{3} B}
$$

where $V_{A_{4}}$ and $V_{A_{3} B}$ are the relaxation interactions, obtained as described above.

We have calculated the local lattice relaxation energy for the same set of $4 d$ and $5 s p$ impurities in $\mathrm{Cu}$. We have used the LDA, which was also the functional used in the FP-KKR Green's function calculations. ${ }^{29}$ In Fig. 5 we compare the results of the ETM model with the corresponding FP-KKR Green's function results. ${ }^{29}$ It is obvious that the agreement between both calculations is very good for the late transition and $s p$ metals, while the relaxation energies for earlier transition metals are substantially overestimated. It is difficult to speculate about the origin of the discrepancy for the relaxation energies in the latter case. One of the reasons could be that the ETM is oversimplified for these systems because of the presence of the multiatom forces due to the open $d$ shell. This will mean that specific local environment effects become important, so that the energetics can no longer be well described as a simple volume effect.

In the figure we have also included the results of the Eshelby theory, ${ }^{31,32}$ according to which

$$
E_{r e l}^{i m p}=\frac{2 B_{B} G_{A}\left(\Omega_{A}-\Omega_{B}\right)^{2}}{3 B_{B} \Omega_{A}+4 G_{A} \Omega_{B}},
$$

where $B_{A}, \Omega_{A}$ and $B_{B}, \Omega_{B}$ are the bulk modulii and equilibrium volume of the host and impurity respectively, and $G_{A}$ the shear modulus of the host. It appears that this theory also works reasonably well, as it should, if the local lattice relaxation effects are indeed a volume effect.

\section{SUMMARY}

We have introduced an effective tetrahedron model for the local lattice relaxations in random metallic alloys and demonstrated that it provides a quantitatively accurate description of the local lattice relaxation energetics in the case of random alloys of the late transition and noble metals. We believe that the success of this model is due to the fact that the local lattice relaxations in these random alloys is a local volume effect. The ETM is very convenient in statistical thermodynamic simulations since the corresponding contribution can be represented in terms of the nearest neighbor pair, triangle, and tetrahedron interactions. The ETM does not, of course, describe inhomogeneous lattice distortions, which should be brought into statistical thermodynamic simulations through another means.

We have also performed ab initio calculations of the local lattice relaxations in $\mathrm{Ni}_{0.50} \mathrm{Pt}_{0.50}$ and $\mathrm{Cu}_{0.75} \mathrm{Au}_{0.25}$ random alloys using 16-, 48-, and 32-atom supercells, respectively. We find that the local lattice relaxation energy is not sensitive to the size of the supercell, which again demonstrates the local character of the phenomenon. Moreover, our relaxation energy for a $\mathrm{Cu}_{0.75} \mathrm{Au}_{0.25}$ alloy obtained in the 32-atom supercell calculations is very close to that found in the eightatom calculations, despite the fact that the average bond length between different alloy components differs significantly.

Finally, using a 32- and 72-atom supercells we have calculated the local lattice relaxations for a single $\mathrm{Cu}$ impurity and a pair of $\mathrm{Cu}$ nearest neighbor atoms in $\mathrm{Au}$. From these calculations we have deduced the average bond length of $\mathrm{Cu}-\mathrm{Au}$ and $\mathrm{Cu}-\mathrm{Cu}$ pairs in the dilute limit of the Au-rich $\mathrm{Cu}-\mathrm{Au}$ alloys. According our results, the $\mathrm{Cu}-\mathrm{Au}$ bond length should be always larger than the average $\mathrm{Cu}-\mathrm{Cu}$ length in random $\mathrm{Cu}-\mathrm{Au}$ alloys. This result is not in contradiction with the existing experimental data. ${ }^{26}$ As far as it concerns the existence of the crossover in the earlier $a b$ initio calculations, ${ }^{2,4}$ we attribute it to the too small size of the supercell used in those calculations.

\section{APPENDIX A: TRANSFORMATION OF THE ETM INTERACTIONS TO THE ISING TYPE HAMILTONIAN}

Let us show how Eq. (2) can be transformed to the usual Ising-type Hamiltonian in the simplest case, when relaxation interactions depend only on the number of $A$ or $B$ atoms in the binary alloy $A_{1-c} B_{c}$. For this purpose we will use the spin-representation in which $E_{r e l}$ (per atom) is determined as

$$
\begin{aligned}
E_{r e l}= & V_{r}^{(0)}+\sum_{i} V_{r}^{(1)} \sigma_{i}+\frac{1}{2} \sum_{i, j}^{\prime} V_{r}^{(2)} \sigma_{i} \sigma_{j} \\
& +\frac{1}{3} \sum_{i, j, k}^{\prime} V_{r}^{(3)} \sigma_{i} \sigma_{j} \sigma_{k}+\frac{1}{4} \sum_{i, j, k, l}^{\prime} V_{r}^{(4)} \sigma_{i} \sigma_{j} \sigma_{k} \sigma_{l} \\
= & \frac{1}{4} \sum_{i, j, k, l} V_{r e l}\left(c_{i}, c_{j}, c_{k}, c_{l}\right),
\end{aligned}
$$


where, $\sigma_{i}=2 c_{i}-1$ is the spin-variable, $V_{r}^{(n)}$ the $n$-site effective relaxation interaction, and $N$ the number of atoms in the system.

In the case of the fcc lattice the one gets the following expressions for the $V_{r}^{(n)}$ by solving this set of equations for some particular chosen tetrahedron with all possible occupation numbers and taking into consideration the number of tetrahedra shared by every geometrical element:

$$
\begin{gathered}
V_{r}^{(0)}=\frac{1}{16}\left(V_{A_{4}}+4 V_{A_{3} B}+6 V_{A_{2} B_{2}}+4 V_{A B_{3}}+V_{B_{4}}\right), \\
V_{r}^{(1)}=-\frac{1}{4}\left(V_{A_{4}}+2 V_{A_{3} B}-2 V_{A B_{3}}-V_{B_{4}}\right), \\
V_{r}^{(2)}=\frac{1}{16}\left(V_{A_{4}}-2 V_{A_{2} B_{2}}+V_{B_{4}}\right), \\
V_{r}^{(3)}=-\frac{1}{32}\left(V_{A_{4}}-2 V_{A_{3} B}+2 V_{A B_{3}}-V_{B_{4}}\right), \\
V_{r}^{(4)}=\frac{1}{32}\left(V_{A_{4}}-4 V_{A_{3} B}+6 V_{A_{2} B_{2}}-4 V_{A B_{3}}+V_{B_{4}}\right) .
\end{gathered}
$$

The first term in Eq. (A1) is just a constant shift and therefore can be neglected. The second term can be also omitted in configurational thermodynamic simulations at a fixed concentration.

\section{APPENDIX B: SPECIAL QUASIRANDOM STRUCTURES}

Special quasirandom structures (SQSs) (Ref. 21) are $\mathrm{N}$-atom per cell periodic structures designed in such a way that their distinct correlation functions $\Pi_{k, m}$ (Ref. 21) best match the ensemble-averaged $\left\langle\Pi_{k, m}\right\rangle$ of the random alloy. Here $(k, m)$ corresponds to the figure defined by the number $\mathrm{k}$ of atoms located on its vertices $(k=2,3,4 \ldots$ are pairs, triangles, tetrahedra, etc.) with $\mathrm{m}$ being the order of neighbor distances separating them $(m=1,2 \ldots$ are first, second neighbors etc.). Obviously, all $\left\langle\Pi_{k, m}\right\rangle$ of the perfectly random binary alloy equal to $(2 x-1)^{k}$, where $x$ is the alloy concentration. The SQSs used in the present work are the following.

\section{SQS-16}

SQS-16 is a supercell designed for $A_{50} B_{50}$ fcc-based alloys and has the space group $C 2 / m$ (space group No. 12 in the International Tables for Crystallography) and a basecentered monoclinic unit cell. Its basis vectors are

$$
\begin{gathered}
\mathbf{a}=\left(\frac{1}{2} \sqrt{\frac{8}{3}},-\frac{1}{2},-\frac{10}{12} \sqrt{3}\right) \eta a, \\
\mathbf{b}=\left(\frac{1}{2} \sqrt{\frac{8}{3}}, \frac{1}{2},-\frac{10}{12} \sqrt{3}\right) \eta a, \\
\mathbf{c}=(0,0, \sqrt{3}) \eta a,
\end{gathered}
$$

where $a$ is the fcc lattice constant and $\eta=\sqrt{2}$. The $A$ atoms lie at sites with Cartesian coordinates (in $\eta a$ units):

$$
\begin{aligned}
A_{1} & =\left(-\sqrt{\frac{3}{8}}, 0, \frac{3}{4} \sqrt{3}\right), \\
A_{2} & =\left(\sqrt{\frac{3}{8}}, 0,-\frac{3}{4} \sqrt{3}\right), \\
A_{3} & =\left(\sqrt{\frac{3}{8}}, 0,-\frac{1}{4} \sqrt{3}\right), \\
A_{4} & =\left(-\sqrt{3} 8,0, \frac{1}{4} \sqrt{3}\right), \\
A_{5} & =\left(-\frac{1}{8} \sqrt{\frac{8}{3}}, \frac{1}{4}, \frac{1}{3} \sqrt{3}\right), \\
A_{6} & =\left(-\frac{1}{8} \sqrt{\frac{8}{3}},-\frac{1}{4}, \frac{1}{3} \sqrt{3}\right), \\
A_{7} & =\left(\frac{1}{8} \sqrt{\frac{8}{3}}, \frac{1}{4},-\frac{1}{3} \sqrt{3}\right), \\
A_{8} & =\left(\frac{1}{8} \sqrt{\frac{8}{3}},-\frac{1}{4},-\frac{1}{3} \sqrt{3}\right) .
\end{aligned}
$$

The $B$ atoms lie at sites with Cartesian coordinates (in $\eta a$ units):

$$
\begin{gathered}
B_{1}=\left(\frac{1}{8} \sqrt{\frac{8}{3}}, 0,-\frac{1}{12} \sqrt{3}\right), \\
B_{2}=\left(-\frac{1}{8} \sqrt{\frac{8}{3}}, 0, \frac{1}{12} \sqrt{3}\right), \\
B_{3}=\left(\frac{1}{8} \sqrt{\frac{8}{3}}, \frac{1}{4}, \frac{1}{6} \sqrt{3}\right), \\
B_{4}=\left(\frac{1}{8} \sqrt{\frac{8}{3}},-\frac{1}{4}, \frac{1}{6} \sqrt{3}\right), \\
B_{5}=\left(-\frac{1}{8} \sqrt{\frac{8}{3}}, \frac{1}{4},-\frac{1}{6} \sqrt{3}\right), \\
B_{6}=\left(-\frac{1}{8} \sqrt{\frac{8}{3}},-\frac{1}{4},-\frac{1}{6} \sqrt{3}\right), \\
B_{7}=\left(-\frac{1}{8} \sqrt{\frac{8}{3}}, 0, \frac{7}{12} \sqrt{3}\right), \\
B_{8}=\left(\frac{1}{8} \sqrt{\frac{8}{3}}, 0,-\frac{7}{12} \sqrt{3}\right) .
\end{gathered}
$$

Several first correlation functions $\Pi_{k, m}$ of SQS-16 are 


$$
\begin{aligned}
& \Pi_{2,1}[6]=0.000, \\
& \Pi_{2,2}[3]=0.000, \\
& \Pi_{2,3}[12]=0.000, \\
& \Pi_{2,4}[6]=0.000, \\
& \Pi_{2,5}[12]=0.000, \\
& \Pi_{2,6}[3]=0.000, \\
& \Pi_{2,7}[18]=0.000, \\
& \Pi_{3,1}[8]=0.000, \\
& \Pi_{3,2}[12]=0.000, \\
& \Pi_{4,1}[2]=0.000, \\
& \Pi_{4,2}[12]=0.000,
\end{aligned}
$$

where the square brackets next to $\Pi_{k, m}$ give the degeneracy factor for the corresponding figure. ${ }^{21}$ Notice that for the perfectly random $A_{50} B_{50}$ alloy all $\left\langle\Pi_{2, m}\right\rangle,\left\langle\Pi_{3, m}\right\rangle$, and $\left\langle\Pi_{4, m}\right\rangle$ are equal to zero.

\section{SQS-48}

SQS-48 is a supercell designed for $A_{50} B_{50}$ fcc-based alloys and has the space group $P 1$ (space group No. 1 in the International Tables for Crystallography) and a triclinic unit cell. Its basis vectors are

$$
\begin{gathered}
\mathbf{a}=\left(\frac{3}{2}, 0,0\right) a, \\
\mathbf{b}=(0,1,0) a, \\
\mathbf{c}=(0,0,1) a .
\end{gathered}
$$

The $A$ atoms lie at sites with Cartesian coordinates (in $a$ units):

$$
\begin{gathered}
A_{1}=\left(0, \frac{1}{4}, \frac{1}{4}\right), \\
A_{2}=\left(-\frac{1}{4}, \frac{1}{4}, 0\right), \\
A_{3}=\left(-\frac{1}{4}, 0, \frac{1}{4}\right), \\
A_{4}=\left(-\frac{1}{2}, \frac{1}{4}, \frac{1}{4}\right), \\
A_{5}=\left(\frac{1}{2}, \frac{1}{4}, \frac{1}{4}\right),
\end{gathered}
$$

$$
\begin{aligned}
& A_{6}=\left(\frac{1}{4}, \frac{1}{4}, 0\right), \\
& A_{7}=\left(\frac{1}{4}, 0, \frac{1}{4}\right),
\end{aligned}
$$$$
A_{8}=\left(-\frac{1}{2}, \frac{1}{2}, 0\right),
$$$$
A_{9}=\left(\frac{1}{2},-\frac{1}{4}, \frac{1}{4}\right),
$$$$
A_{10}=\left(\frac{1}{4},-\frac{1}{4}, 0\right),
$$$$
A_{11}=\left(0, \frac{1}{4},-\frac{1}{4}\right),
$$$$
A_{12}=\left(-\frac{1}{4}, \frac{1}{4}, \frac{1}{2}\right) \text {, }
$$

$A_{13}=\left(-\frac{1}{4}, 0,-\frac{1}{4}\right)$,

$A_{14}=\left(-\frac{1}{2}, \frac{1}{4},-\frac{1}{4}\right)$,

$$
A_{15}=\left(\frac{3}{4}, 0,-\frac{1}{4}\right),
$$

$$
A_{16}=\left(\frac{1}{2}, 0, \frac{1}{2}\right),
$$

$$
A_{17}=\left(\frac{1}{4}, \frac{1}{4}, \frac{1}{2}\right),
$$

$$
A_{18}=\left(\frac{1}{4}, 0,-\frac{1}{4}\right),
$$

$$
A_{19}=\left(0, \frac{1}{2}, \frac{1}{2}\right),
$$

$A_{20}=\left(0,-\frac{1}{4},-\frac{1}{4}\right)$,

$A_{21}=\left(-\frac{1}{4},-\frac{1}{4}, \frac{1}{2}\right)$,

$A_{22}=\left(-\frac{1}{4}, \frac{1}{2},-\frac{1}{4}\right)$,

$A_{23}=\left(\frac{3}{4},-\frac{1}{4}, \frac{1}{2}\right)$, 


$$
A_{24}=\left(\frac{1}{4}, \frac{1}{2},-\frac{1}{4}\right)
$$

The $B$ atoms lie at sites with Cartesian coordinates (in $a$ units)

$$
\begin{gathered}
B_{1}=(0,0,0), \\
B_{2}=\left(-\frac{1}{2}, 0,0\right), \\
B_{3}=\left(\frac{3}{4}, \frac{1}{4}, 0\right), \\
B_{16}=\left(\frac{3}{4}, 0, \frac{1}{4}\right), \\
B_{14}=\left(\frac{1}{4}, \frac{1}{2}, \frac{1}{4}\right), \\
B_{13}=\left(\frac{1}{2}, 0,0\right), \\
B_{12}=\left(\frac{3}{2}, \frac{1}{2}, \frac{1}{2}, \frac{1}{4}\right), \\
B_{10}=\left(\frac{1}{4}, \frac{1}{4},-\frac{1}{2}\right), \\
B_{7}=\left(\frac{1}{2}, 0\right), \\
B_{8}=\left(\frac{1}{4}, \frac{1}{2}, \frac{1}{2}, \frac{1}{4},-\frac{1}{4}, \frac{1}{4}, \frac{1}{4}\right), \\
\left.-\frac{1}{4}\right),
\end{gathered}
$$

$$
\begin{gathered}
B_{18}=\left(\frac{1}{2}, \frac{1}{4},-\frac{1}{4}\right), \\
B_{19}=\left(-\frac{1}{2}, \frac{1}{2}, \frac{1}{2}\right), \\
B_{20}=\left(-\frac{1}{2},-\frac{1}{4},-\frac{1}{4}\right), \\
B_{21}=\left(\frac{3}{4}, \frac{1}{2},-\frac{1}{4}\right), \\
B_{22}=\left(\frac{1}{2}, \frac{1}{2}, \frac{1}{2}\right), \\
B_{23}=\left(\frac{1}{2},-\frac{1}{4},-\frac{1}{4}\right), \\
B_{24}=\left(\frac{1}{4},-\frac{1}{4}, \frac{1}{2}\right) .
\end{gathered}
$$

Several first correlation functions $\Pi_{k, m}$ of SQS-48 are

$$
\begin{gathered}
\Pi_{2,1}[6]=0.000, \\
\Pi_{2,2}[3]=0.000 \\
\Pi_{2,3}[12]=0.000, \\
\Pi_{2,4}[6]=0.000, \\
\Pi_{2,5}[12]=0.000 \\
\Pi_{2,6}[3]=-0.083, \\
\Pi_{2,7}[18]=-0.056 \\
\Pi_{3,1}[8]=0.000 \\
\Pi_{3,2}[12]=-0.056 \\
\Pi_{4,1}[2]=-0.167, \\
\Pi_{4,2}[12]=-0.056
\end{gathered}
$$

\section{SQS-32}

SQS-32 is a supercell designed for $A_{75} B_{25}$ fcc-based alloys and has the space group $P 1$ (space group No. 1 in the International Tables for Crystallography) and a triclinic unit cell. Its basis vectors are

$$
\begin{gathered}
\mathbf{a}=\left(\sqrt{\frac{8}{3}}, 0,-\frac{1}{3}\right) \eta a, \\
\mathbf{b}=(0,1,0) \eta a, \\
\mathbf{c}=(0,0, \sqrt{3}) \eta a,
\end{gathered}
$$


where $a$ is the fcc lattice constant and $\eta=\sqrt{2}$. The $A$ atoms lie at sites with Cartesian coordinates (in $\eta a$ units):

$$
\begin{aligned}
& A_{1}=(0,0,0), \\
& A_{2}=\left(0, \frac{1}{2}, 0\right) \text {, } \\
& A_{3}=\left(\frac{1}{2} \sqrt{\frac{8}{3}}, 0,-\frac{1}{6} \sqrt{3}\right), \\
& A_{4}=\left(-\frac{1}{4} \sqrt{\frac{8}{3}}, \frac{1}{4}, \frac{1}{12} \sqrt{3}\right) \text {, } \\
& A_{5}=\left(-\frac{1}{4} \sqrt{\frac{8}{3}},-\frac{1}{4}, \frac{1}{12} \sqrt{3}\right) \text {, } \\
& A_{6}=\left(0,-\frac{1}{4},-\frac{1}{4} \sqrt{3}\right) \text {, } \\
& A_{7}=\left(0, \frac{1}{4},-\frac{1}{4} \sqrt{3}\right), \\
& A_{8}=\left(\frac{1}{4} \sqrt{\frac{8}{3}}, \frac{1}{2},-\frac{1}{3} \sqrt{3}\right), \\
& A_{9}=\left(\frac{1}{4} \sqrt{\frac{8}{3}}, 0,-\frac{1}{3} \sqrt{3}\right), \\
& A_{10}=\left(\frac{1}{2} \sqrt{\frac{8}{3}},-\frac{1}{4},-\frac{5}{12} \sqrt{3}\right) \text {, } \\
& A_{11}=\left(-\frac{1}{4} \sqrt{\frac{8}{3}}, 0,-\frac{1}{6} \sqrt{3}\right), \\
& A_{12}=\left(-\frac{1}{4} \sqrt{\frac{8}{3}}, \frac{1}{2},-\frac{1}{6} \sqrt{3}\right) \text {, } \\
& A_{13}=\left(0, \frac{1}{2}, \frac{1}{2} \sqrt{3}\right), \\
& A_{14}=\left(\frac{1}{4} \sqrt{\frac{8}{3}}, \frac{1}{4}, \frac{5}{12} \sqrt{3}\right), \\
& A_{15}=\left(\frac{1}{4} \sqrt{\frac{8}{3}},-\frac{1}{4}, \frac{5}{12} \sqrt{3}\right), \\
& A_{16}=\left(\frac{1}{2} \sqrt{\frac{8}{3}}, 0, \frac{1}{3} \sqrt{3}\right), \\
& A_{17}=\left(\frac{1}{2} \sqrt{\frac{8}{3}}, \frac{1}{2}, \frac{1}{3} \sqrt{3}\right), \\
& A_{18}=\left(-\frac{1}{4} \sqrt{\frac{8}{3}},-\frac{1}{4}, \frac{7}{12} \sqrt{3}\right) \text {, }
\end{aligned}
$$

$$
\begin{gathered}
A_{19}=\left(-\frac{1}{4} \sqrt{\frac{8}{3}}, \frac{1}{4}, \frac{7}{12} \sqrt{3}\right), \\
A_{20}=\left(0, \frac{1}{4}, \frac{1}{4} \sqrt{3}\right), \\
A_{21}=\left(0,-\frac{1}{4}, \frac{1}{4} \sqrt{3}\right), \\
A_{22}=\left(\frac{1}{4} \sqrt{\frac{8}{3}}, 0, \frac{1}{6} \sqrt{3}\right), \\
A_{23}=\left(\frac{1}{2} \sqrt{\frac{8}{3}},-\frac{1}{4}, \frac{1}{12} \sqrt{3}\right), \\
A_{24}=\left(-\frac{1}{4} \sqrt{\frac{8}{3}}, \frac{1}{2}, \frac{1}{3} \sqrt{3}\right) .
\end{gathered}
$$

The $B$ atoms lie at sites with Cartesian coordinates (in $\eta a$ units):

$$
\begin{aligned}
B_{1} & =\left(\frac{1}{4} \sqrt{\frac{8}{3}},-\frac{1}{4},-\frac{1}{12} \sqrt{3}\right), \\
B_{2} & =\left(\frac{1}{4} \sqrt{\frac{8}{3}}, \frac{1}{4},-\frac{1}{12} \sqrt{3}\right), \\
B_{3} & =\left(\frac{1}{2} \sqrt{\frac{8}{3}}, \frac{1}{2},-\frac{1}{6} \sqrt{3}\right), \\
B_{4} & =\left(\frac{1}{2} \sqrt{\frac{8}{3}}, \frac{1}{4},-\frac{5}{12} \sqrt{3}\right), \\
B_{6} & =\left(\frac{1}{4} \sqrt{\frac{8}{3}}, \frac{1}{2}, \frac{1}{6} \sqrt{3}\right), \\
B_{7} & =\left(\frac{1}{2} \sqrt{\frac{8}{3}}, \frac{1}{4}, \frac{1}{12} \sqrt{3}\right), \\
B_{8} & =\left(-\frac{1}{3} \sqrt{\frac{8}{3}}, 0, \frac{1}{3} \sqrt{3}\right) .
\end{aligned}
$$

Several first correlation functions $\Pi_{k, m}$ of SQS-32 are

$$
\begin{aligned}
& \Pi_{2,1}[6]=0.250, \\
& \Pi_{2,2}[3]=0.250, \\
& \Pi_{2,3}[12]=0.229, \\
& \Pi_{2,4}[6]=0.250, \\
& \Pi_{2,5}[12]=0.250,
\end{aligned}
$$




$$
\begin{gathered}
\Pi_{2,6}[3]=0.286, \\
\Pi_{2,7}[18]=0.244, \\
\Pi_{3,1}[8]=0.125, \\
\Pi_{3,2}[12]=0.0625,
\end{gathered}
$$

$$
\begin{gathered}
\Pi_{4,1}[2]=0.000, \\
\Pi_{4,2}[12]=0.0417 .
\end{gathered}
$$

Notice that for the perfectly random $A_{75} B_{25}$ alloy all $\left\langle\Pi_{2, m}\right\rangle=0.25$, all $\left\langle\Pi_{3, m}\right\rangle=0.125$, and all $\left\langle\Pi_{4, m}\right\rangle=0.0625$.
${ }^{1}$ D. G. Pettifor, Bonding and Structure of Molecules and Solids (Clarendon Press, Oxford, 1995).

${ }^{2}$ V. Ozolins, C. Wolverton, and A. Zunger, Phys. Rev. B 57, 6427 (1998).

${ }^{3}$ A. V. Ruban, I. A. Abrikosov, and H. L. Skriver, Phys. Rev. B 51, 12958 (1995).

${ }^{4}$ L. G. Ferreira, V. Ozolins, and A. Zunger, Phys. Rev. B 60, 1687 (1999).

${ }^{5}$ M. A. Krivoglaz, Diffraction of X-Rays and Neutrons in Imperfect Crystals (Springer, Berlin, 1991); M. A. Krivoglaz, Diffuse Scattering of X-Rays and Neutrons by Fluctuations (Springer, Berlin, 1996).

${ }^{6}$ A. G. Khachaturyan, Theory of Structural Transformations in Solids (Wiley, New York, 1983).

${ }^{7}$ C. Amador, W. R. L. Lambrecht, M. van Schilfgaarde, and B. Segal, Phys. Rev. B 47, 15276 (1993); C. Amador and G. Bozzolo, ibid. 49, 956 (1994).

${ }^{8}$ J. S. Faulkner, Prog. Mater. Sci. 27, 1 (1982).

${ }^{9}$ B. L. Györffy, Phys. Rev. B 5, 2382 (1972).

${ }^{10}$ F. Ducastelle, Order and Phase Stability in Alloys (NorthHolland, Amsterdam, 1991).

${ }^{11}$ A. V. Ruban and H. L. Skriver, Comput. Mater. Sci. 15, 119 (1999).

${ }^{12}$ N. E. Christensen and S. Satpathy, Phys. Rev. Lett. 55, 600 (1985).

${ }^{13}$ J. P. Perdew and Y. Wang, Phys. Rev. B 45, 13244 (1992).

${ }^{14}$ L. Vitos, B. Johansson, J. Kollar, and H. L. Skriver, Phys. Rev. A 61, 52511 (2000); L. Vitos, B. Johansson, J. Kollar, and H. L. Skriver, Phys. Rev. B 62, 10046 (2000).

${ }^{15}$ Y. Wang and J. P. Perdew, Phys. Rev. B 44, 13298 (1991); J. P. Perdew, J. A. Chevary, S. H. Vosko, K. A. Jackson, M. R. Pederson, D. J. Singh, and C. Fiolhais, ibid. 46, 6671 (1992).
${ }^{16}$ A. V. Ruban and H. L. Skriver, Phys. Rev. B 66, 024201 (2002); A. V. Ruban, S. I. Simak, P. A. Korzhavyi, and H. L. Skriver, ibid. 66, 024202 (2002).

${ }^{17}$ I. A. Abrikosov, A. M. N. Niklasson, S. I. Simak, B. Johansson, A. V. Ruban, and H. L. Skriver, Phys. Rev. Lett. 76, 4203 (1996); I. A. Abrikosov, S. I. Simak, B. Johansson, A.V. Ruban, and H. L. Skriver, Phys. Rev. B 56, 9319 (1997).

${ }^{18}$ A. Khein, D. J. Singh, and C. J. Umrigar, Phys. Rev. B 51, 4105 (1995).

${ }^{19}$ G. Kresse and J. Hafner, Phys. Rev. B 48, 13115 (1993).

${ }^{20}$ G. Kresse and J. Furthmüller, Comput. Mater. Sci. 6, 15 (1996); Phys. Rev. B 54, 11169 (1996).

${ }^{21}$ A. Zunger, S.-H. Wei, L. G. Ferreira, and J. E. Bernard, Phys. Rev. Lett. 65, 353 (1990).

${ }^{22}$ D. Vanderbilt, Phys. Rev. B 41, 7892 (1990).

${ }^{23}$ G. Kresse and J. Joubert, Phys. Rev. B 59, 1758 (1999); P. E. Blöchl, ibid. 50, 17953 (1994).

${ }^{24}$ H. J. Monkhorst and J. D. Pack, Phys. Rev. B 13, 5188 (1972).

${ }^{25}$ Z. W. Lu, S.-H. Wei, and Z. Zunger, Europhys. Lett. 21, 221 (1993).

${ }^{26}$ A. I. Frenkel, E. A. Stern, A. Rubshtein, A. Voronel, and Yu. Rosenberg, J. Phys. IV 7, 1005 (1997).

${ }^{27}$ O. Malis, K. F. Ludwig, Jr., D. L. Olmsted, and B. Chakraborty, Philos. Mag. B 79, 869 (1999).

${ }^{28}$ A. Christensen, A. V. Ruban, P. Sotlze, K. W. Jacobsen, H. L. Skriver, and J. K. Norskov, Phys. Rev. B 56, 5822 (1997).

${ }^{29}$ N. Papanikolaou, R. Zeller, P. H. Dederichs, and N. Stefanou, Phys. Rev. B 55, 4157 (1997).

${ }^{30}$ A. V. Ruban and H. L. Skriver, Phys. Rev. B 55, 8801 (1997).

${ }^{31}$ J. D. Eshelby, Solid State Phys. 3, 79 (1956).

${ }^{32}$ A. K. Niessen and A. R. Miedema, Ber. Bunsenges. Phys. Chem. 87, 717 (1983). 